\title{
Saturation of two-photon excitation provides insight into the effects of a quantum dot blinking suppressant: A fluorescence correlation spectroscopy study \\ Romey F Heuff ${ }^{l}$, Michele Marrocco ${ }^{2}$ and David T Cramb ${ }^{1}$ *
}

Supplementary Information

Below are the data used to determine the two-photon excitation fluorescence saturation parameters from equation 3 in the main text. The fits to the data (black lines) work are reasonable with the exception of the red (605 nm emission) quantum dots after being exposed to BME (yellow triangle, panel B).
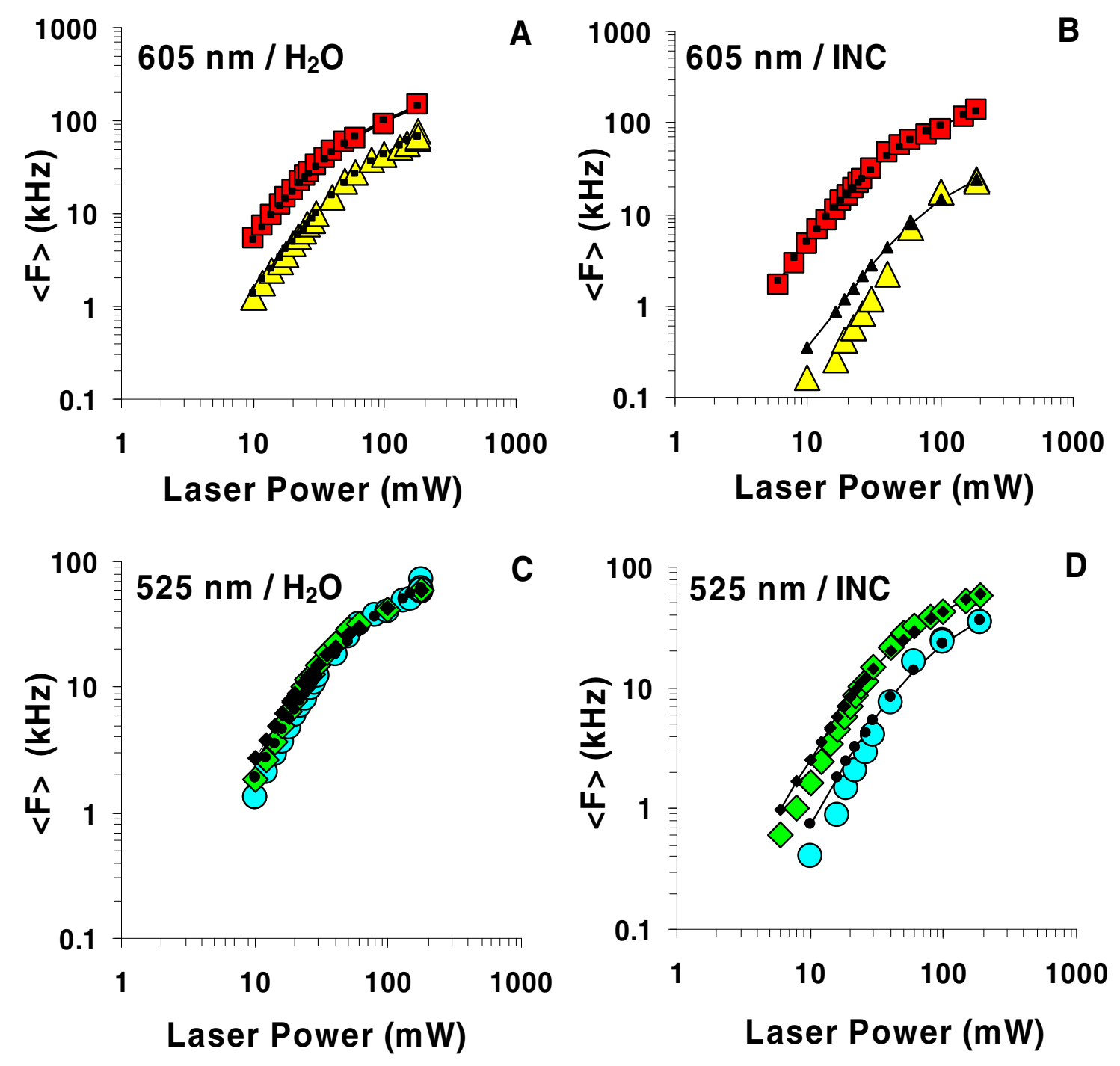
Figure S1: Determination of saturation threshold powers using Equation 2 before and after the addition of BME for $605 \mathrm{~nm}$ and $525 \mathrm{~nm}$ carboxylated CdSe/ZnS quantum dots in incubation buffer (INC) and ultrapure water $\left(\mathbf{H}_{2} \mathbf{O}\right)$. (A) and (B) The $605 \mathrm{~nm}$ data are shown as red squares (before) and yellow triangles (after) the addition of $5 \mathrm{ul}$ of neat $\beta$-mercaptoethanol (BME) to give a final concentration of BME of $0.32 \mathrm{mM}$. (C) and (D) The $525 \mathrm{~nm}$ data are given as green diamonds (before) and blue circles (after) the addition of the same amount of BME. The fitting results are the black lines / labels. 